\title{
Prototyping the central control system for the Cherenkov Telescope Array
}

D. Melkumyan, I. Oya, J. Bobnar, S. Sah, R. Anutarawiramkul, et al.

D. Melkumyan, I. Oya, J. Bobnar, S. Sah, R. Anutarawiramkul, M. Füßling, I. Sadeh, M. Sekoranja, T. Schmidt, U. Schwanke, J. Schwarz, G. Spengler, P. Wegner, "Prototyping the central control system for the Cherenkov Telescope Array," Proc. SPIE 10707, Software and Cyberinfrastructure for Astronomy V, 107071R (6 July 2018); doi: $10.1117 / 12.2309659$

Event: SPIE Astronomical Telescopes + Instrumentation, 2018, Austin, Texas, United States 


\title{
Prototyping the central control system for the Cherenkov Telescope Array
}

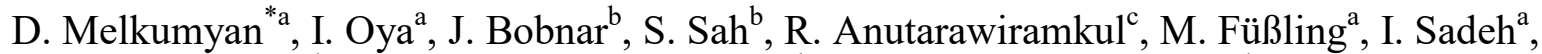 \\ M. Sekoranja ${ }^{\text {b }}$, T. Schmidt ${ }^{\mathrm{a}}$, U. Schwanke ${ }^{\mathrm{d}}$, J. Schwarz ${ }^{\mathrm{e}}$, G. Spengler ${ }^{\mathrm{d}}$, P. Wegner $^{\mathrm{a}}$ \\ for the CTA Consortium ${ }^{\mathrm{f}}$ \\ ${ }^{a}$ DESY, Platanenallee 6, D-15738 Zeuthen, Germany, \\ ${ }^{\mathrm{b}}$ Cosylab d.d., Gerbičeva ulica 64, SL-1000 Ljubljana, Slovenia, \\ ${ }^{c}$ National Astronomical Research Institute of Thailand, A. Mae Rim, Chiang Mai 50180, Thailand, \\ ${ }^{\mathrm{d}}$ Institut für Physik, Humboldt-Universität zu Berlin, Newtonstraße 15, 12489 Berlin, Germany, \\ ${ }^{e}$ INAF - Osservatorio Astronomico di Brera, Via E. Bianchi, 46, 23807 San Rocco LC, Italy, \\ ${ }^{\mathrm{f}}$ Full consortium author list at http://cta-observatory.org
}

\begin{abstract}
The Cherenkov Telescope Array (CTA) will be the next generation ground-based observatory for gamma-ray astronomy at very-high energies. CTA will consist of two large arrays with 118 Cherenkov telescopes in total, deployed in the northern and southern hemispheres. The Observation Execution System (OES) provides the means to execute observations and to handle the acquisition of scientific data in CTA. The Manager and Central Control (MCC) system is a core element in the OES system that implements the execution of observation requests received from the scheduler sub-system. This contribution provides a summary of the main MCC design features and of the plans for prototyping.
\end{abstract}

Keywords: CTA, OES, MCC, Cherenkov Telescope Array, Central Control, Resource Manager

\section{INTRODUCTION}

The Cherenkov Telescope Array (CTA) ${ }^{1}$ is the next generation observatory for ground-based very-high-energy gammaray astronomy. In order to provide full sky coverage, CTA will consist of two large arrays with 118 Cherenkov telescopes in total, to be deployed in the Northern (La Palma, Spain) and Southern (Paranal, Chile) Hemispheres. The Southern Hemisphere array will span the entire energy range of CTA, covering gamma-ray energies from $\sim 20 \mathrm{GeV}$ to $\sim 300 \mathrm{TeV}$, mainly dedicated to Galactic sources. The northern hemisphere array will focus on low- and mid-energy ranges from $\sim 20 \mathrm{GeV}$ to $\sim 5 \mathrm{TeV}$, mainly dedicated to extragalactic objects. In addition to a large number of scientific instruments, CTA will comprise three types of telescopes covering different energy ranges: Small Sized Telescopes $(\sim 4$ $\mathrm{m}$ diameter) to cover the highest energy gamma-rays, Medium Sized Telescopes $(\sim 12 \mathrm{~m})$ to cover the core energy range of CTA and Large Sized Telescopes ( $\sim 23 \mathrm{~m}$ ) sensitive to low-energy gamma-rays (SSTs, MSTs and LSTs, respectively).

The Observation Execution System (OES) provides the means to execute observations and to handle the acquisition of scientific data in CTA.

In this paper, we focus on the design and implementation of one of the top-level sub-systems of the OES, the Manager and Central Control (MCC) system. The purpose of MCC is to execute observations requests received from the ShortTerm Scheduler sub-system and to provide infrastructure services concerning the administration of various resources to all OES sub-systems.

This paper is organized as follows: Section 2 briefly describes the OES system. Section 3 gives an overview on the MCC sub-system. An implementation detail of the Manager and the Central Control components is given in Sections 4 and 5, respectively. Finally, section 5 presents our conclusions and future tasks.

*david.melkumyan@desy.de

Software and Cyberinfrastructure for Astronomy V, edited by Juan C. Guzman, Jorge Ibsen, Proc. of SPIE Vol. 10707, 107071R · @ 2018 SPIE · CCC code: 0277-786X/18/\$18 · doi: 10.1117/12.2309659 


\section{OBSERVATION EXECUTION SYSTEM}

OES will provide the functionality required to monitor and control all telescopes and auxiliary instruments in CTA; to prepare, schedule and perform observations and calibration procedure; to time-stamp, read-out, filter and store data; and to produce status and quality reports.

The OES architecture was designed using the Software Platform Embedded System (SPES) ${ }^{2}$ methodology. A combination of OMG systems Modeling Language ${ }^{\dagger}$ (SysML) and Unified Modeling Language (UML) was employed to model the OES system architecture ${ }^{3}$ (system requirements, system behaviors and sub-systems). The OES system is composed of several closely interrelated sub-systems (Short-Term Scheduler and ToO Handler, Manager and Central Control, Data Handling System, Operator Interface, Monitoring, Reporting and Diagnosis). These sub-systems are split up further into individual components. Each sub-system and each component serves a well-defined easily comprehensible purpose. This architectural approach allows us to implement a flexible use-case driven software development approach thanks to the traceability from use cases to the logical software elements.

The OES will be implemented as distributed software system using the ALMA Common Software (ACS $)^{4,5}$, which is a set of application frameworks built on top of CORBA middleware. ACS is based on a container-component model and supports the programming languages $\mathrm{C}++$, Java and Python. Each CTA site will contain one instance of the OES system.

\section{MANAGER AND CENTRAL CONTROL SYSTEM}

MCC is one of the top-level sub-systems of the OES. It was prototyped following the Model-Driven Architecture (MDA) approach of $\mathrm{OES}^{6}$. MCC is comprised of two components; namely, Manager and Central Control. Figure 1 shows a UML diagram indicating the main components of the MCC sub-system.

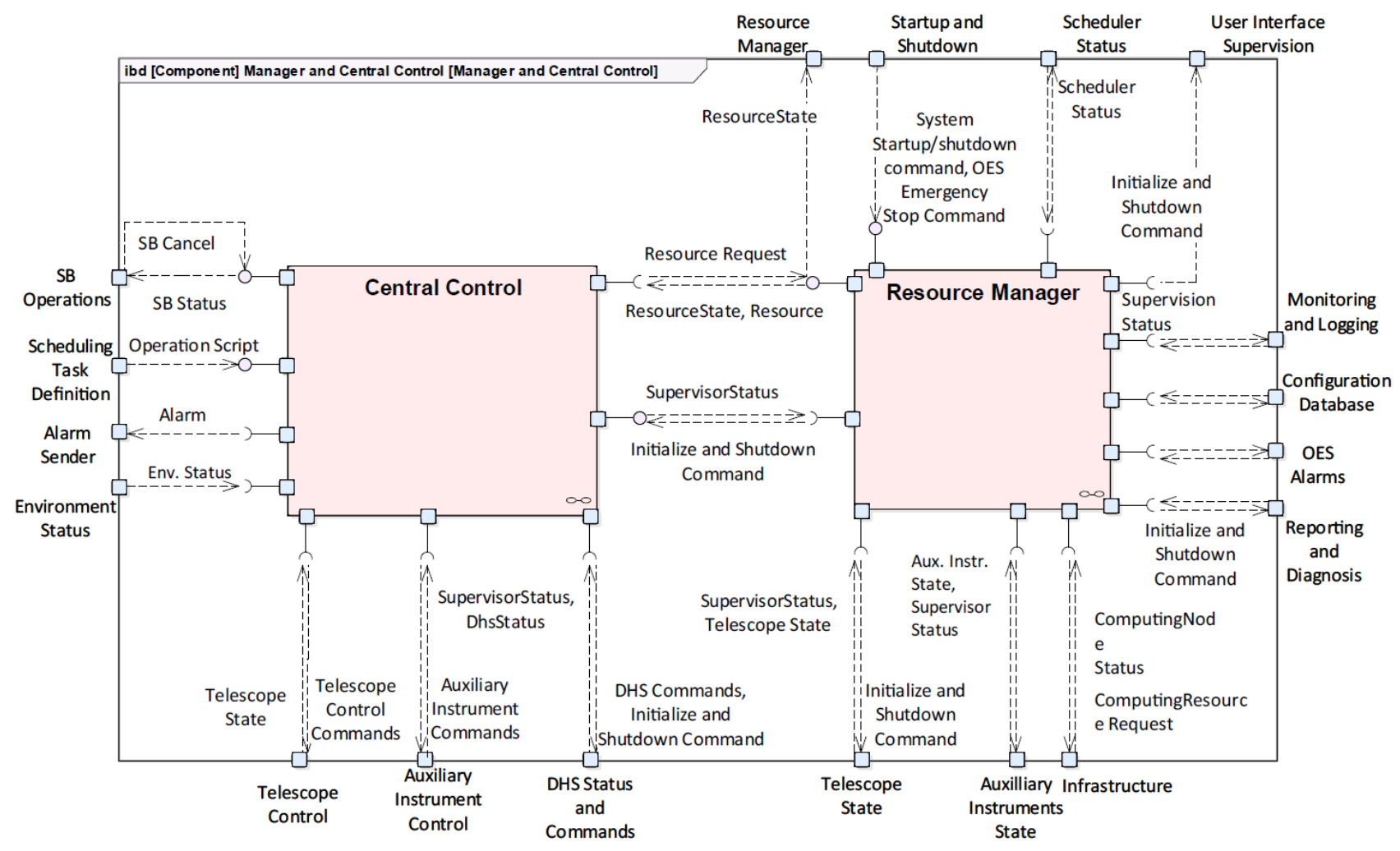

Figure 1. Logical view of MCC, representing the highest-level components and the most relevant data elements and interfaces of the system.

\footnotetext{
${ }^{\dagger}$ SysML - "OMG Systems Modeling Language 1.4", OMG 2015, https://www.omg.org/spec/SysML/1.4/

‡ OMG UML - "OMG Formal Versions of UML",OMG 2017, https://www.omg.org/spec/UML/2.5.1/
} 
The Manager component is the master element that provides the OES system with start-up, shut-down, supervision and registry functionalities.

The Central Control component is responsible for execution of scheduling blocks (SB) received from the Short-Term Scheduler $^{7}$ (Scheduler). It defines sub-arrays and sends corresponding commands to the telescopes, auxiliary instruments and to the Data Handling System ${ }^{8}$ (DHS). The CC then monitors the observation progress and provides this information to the Operator User Interface 9 (UI). One instance of MCC exists per OES system.

\section{RESOURCE MANAGER}

The Resource Manager (RM) provides administrative and infrastructure services concerning various resources to all OES sub-systems. The administered resources comprise telescopes, auxiliary instruments, computing nodes and OES components. The Scheduler and the RM share responsibility regarding the management of telescopes. A telescope that should not be scheduled for sub-array actions can be marked unavailable in the RM via the operator UI. RM and Scheduler cooperate: on the one hand, on any change of telescope availability, the RM informs the schedulers; on the other hand, at the beginning of every short-term scheduler run, the Scheduler asks the RM for current telescope availability information. Any unavailable telescopes will not be used by the schedule that is being created. Other resources (e.g. computing nodes) are entirely managed by the RM.

\subsection{Supervision Tree}

Reliable operation is one main quality requirement of OES. However, failure of software components can never be excluded entirely. To handle potential failure, OES employs a supervision tree concept, used in Erlang's fault management system ${ }^{\S}$. For that purpose, the system is structured as a tree, where each tree node supervising its child nodes. The supervision includes the following functions: starting a supervised component; monitoring the status of a supervised component, and replacing a supervised component if something goes wrong. Some special treatment is provided in leaf components that are statically deployed close to a particular resource (e.g. telescopes). Figure 2 shows a UML sequence diagram that illustrates the behavior of instantiation and supervision of one of the "mid-level" components (i.e. Sub-array Supervisor).

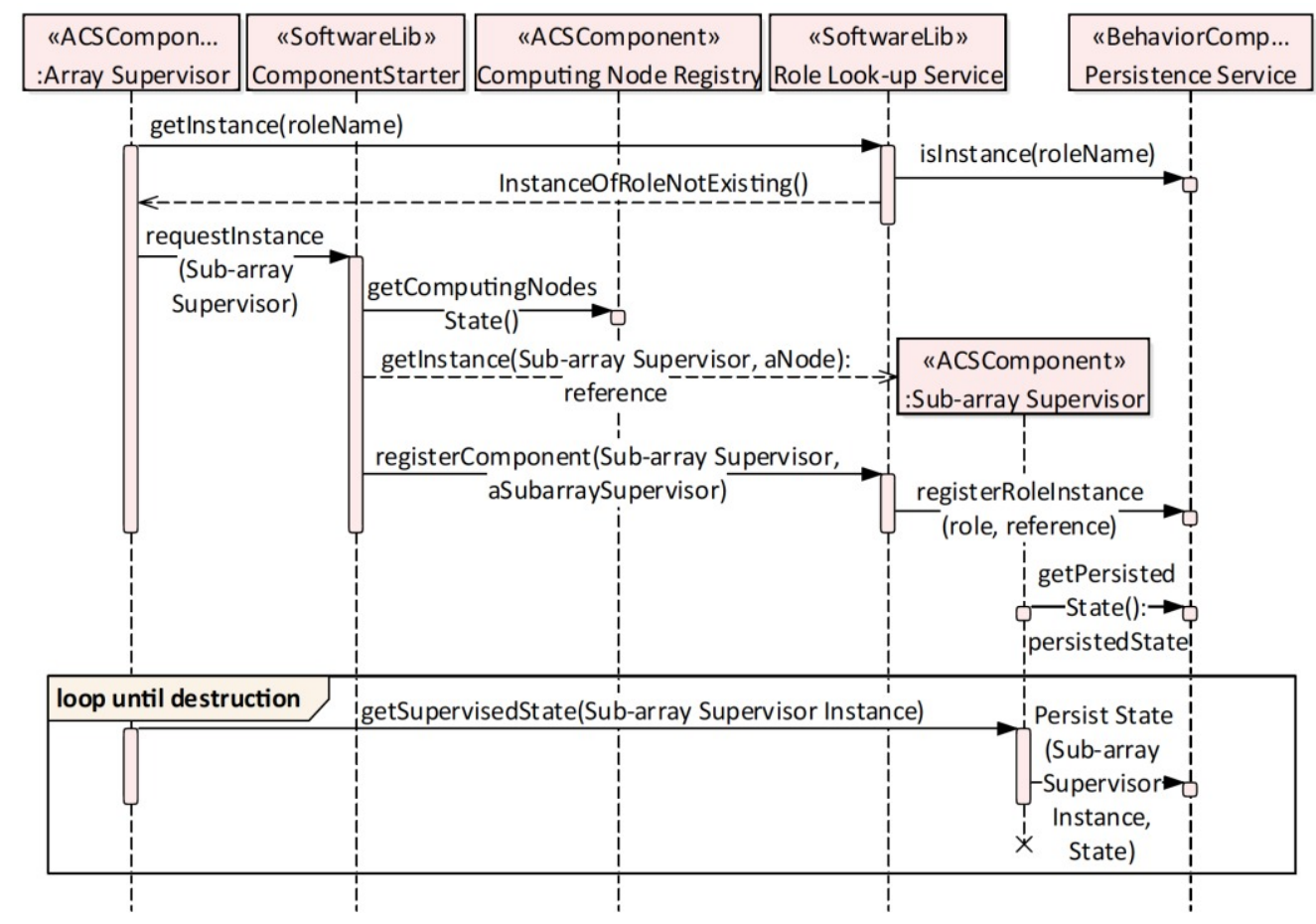

Figure 2. UML sequence diagram that illustrates the behavior of a component's instantiation and supervision.

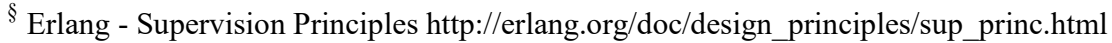




\subsection{Control Tickets}

In the supervisor tree, the life time of a predecessor and the corresponding successor component might overlap. For the conflict avoidance, the API methods of each service component will include a control ticket. The latter will be used to guard against conflicting concurrent access, where only a single control ticket can be valid at a time (i.e. control tickets constructed later invalidate those constructed earlier). The client should then operate with the particular component instance which owns the valid ticket. All commands that change state inside the OES command tree have to carry a control ticket. Control tickets are not required for read access, which does not change state. Figure 3 shows the logical view of the Resource Manager. Only the highest-level components and the most relevant data elements and interfaces are shown.

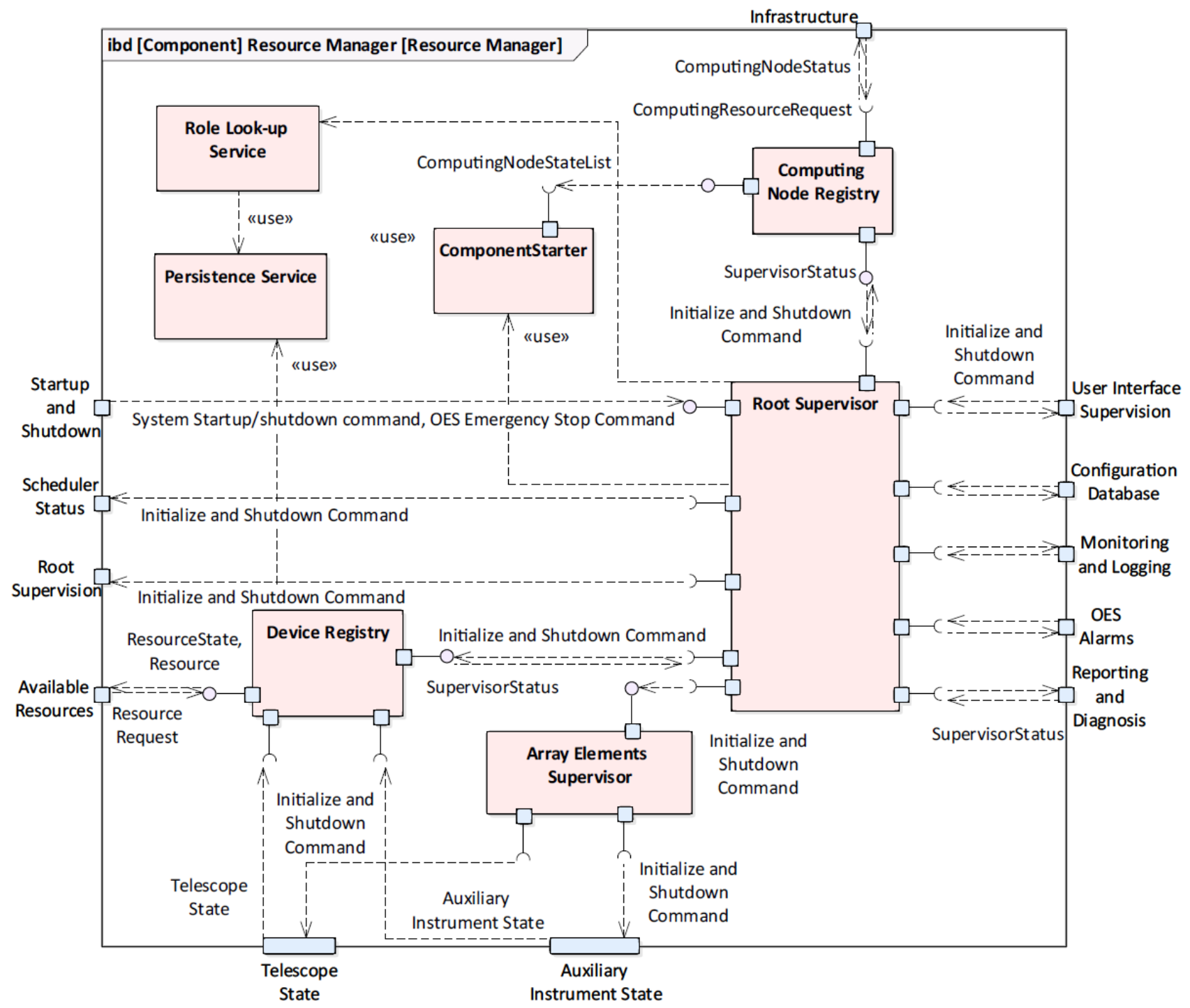

Figure 3.The logical view of the Resource Manager. Only the highest-level components, and the most relevant data elements and interfaces are shown.

The Resource Manager consists of the following components:

- Root Supervisor (Java ACS component): The Root Supervisor is the root node of the OES supervision tree. All components started by the root supervisor are deployed to run "always", and are restarted by the supervisor in case of a problem. For fault tolerance, the Root Supervisor component is instantiated in two instances ("cold" 
and "hot" instances), deployed on different computing nodes. The list of all components to be supervised by the Root Supervisor is statically configured through the ACS configuration database.

- Component Starter (Java library): The Component Starter provides services to any supervisor component that needs to instantiate and start another component. The Component Starter is capable of finding a computing node appropriate for hosting a particular supervised component to be started. It helps a supervisor component to get it up and running, providing pertinent run-time initialization parameters in the process, which may be component-specific.

- Device Registry (Java ACS component): The Device Registry tracks availability for service of telescopes and auxiliary devices. If some device has been taken out of service because of problems, maintenance, or other reasons, this information is stored in the Device Registry.

- Computing Node Registry (Java ACS component): The Computing Node Registry tracks availability and utilization of computing nodes used by OES. It can be used by the operator UI to obtain a list of all nodes utilized by OES components; to check individual availability and idle status; and to take some computing nodes out of service or re-activate them.

- Persistence Service (Java library): The Persistence Service is an OES-wide service to persist the state of those components that require it. A simple-to-use development version of the Persistence Service is implemented. This development version reads its content from a flat file in JSON format.

- Role Look-up Service (Java library): The Role Look-up Service allows look-up of components by role name, facilitating identification of a service component by functionality. It provides convenient access to the currently active member of the successor chain of an OES component. The Role Look-up Service also keeps references of all instances of all components (acting with some role), as well as the particular control ticket each one holds. Almost every OES component uses the role look-up functionality of the Resource Manager.

- Array Elements Supervisor: The Array Elements Supervisor provides the capability to startup, shutdown, and supervise software components of Array Elements.

\section{CENTRAL CONTROL}

The Central Control component implements the execution of scheduling blocks received from the Scheduler sub-system. $\mathrm{CC}$ uses the monitoring sub-system to oversee the conditions to continue the execution of a SB, and cancels that execution if the conditions become adverse. Figure 4 shows the logical view of the Central Control. Only the highestlevel components and the most relevant data elements and interfaces are shown.

$\mathrm{CC}$ consists of the following components:

- Array Supervisor (Java ACS component): One Array Supervisor exists only once per site. Receiving new sub-array actions from the Scheduler causes the array supervisor to respond, instantiating as needed any subarray supervisors. When creating the Sub-array Supervisor, the Array Supervisor hands over run-time information such as the list of telescopes allocated to the sub-array to act on. The Array Supervisor is supervised by the Root Supervisor of the Resource Manager. The Array Supervisor provides SB execution statuses to other sub-systems; in particular, it informs the Scheduler when an observation has been completed.

- Sub-array Supervisor (Java ACS component): The Sub-array Supervisor receives from the Array Supervisor a list of telescopes and a sub-array action to execute on those telescopes. Given the sub-array action type, the Sub-array Supervisor has the intelligence to determine which sub-array sequencer to instantiate. It derives this from an identifier in the SB. After initialization, the Sub-array Supervisor asks the Sub-array Sequencer whether any additional components (e.g. DHS) need to be initialized and supervised. The Sub-array Supervisor also checks that the conditions associated to that sub-array, such as good weather conditions or allocation of telescopes are being fulfilled. These conditions are specified in the SB. Whenever one of them fails to be met, the observations are stopped. The Sub-array Supervisor informs the Array Supervisor when processing of the corresponding sub-array action has come to an end. 


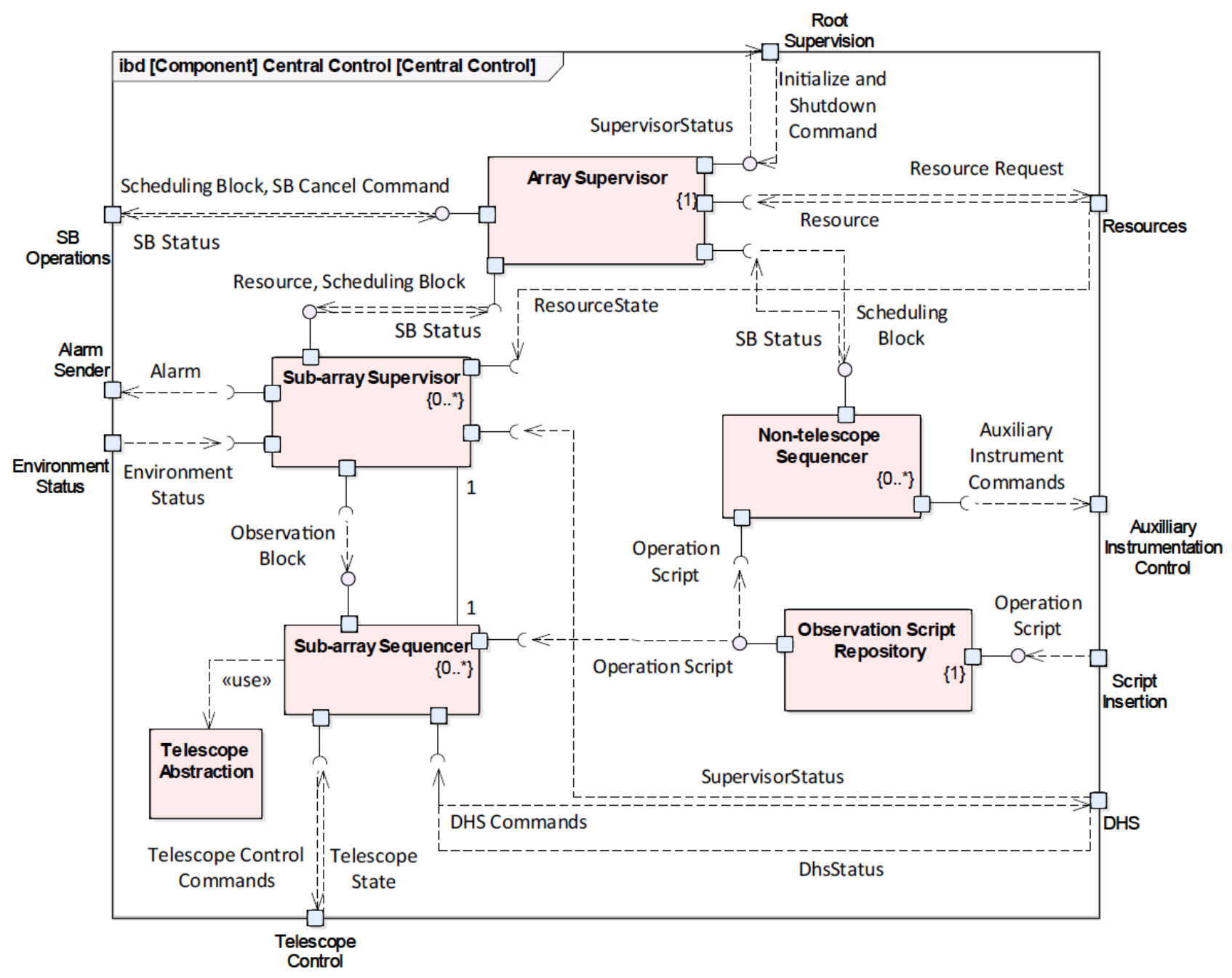

Figure 4. Diagram showing the logical view of the Central Control. Only the highest-level components, and the most relevant data elements and interfaces are shown.

- Sub-array Sequencer (Python ACS component): One Sub-array Sequencer is responsible for the execution of one sub-array action, as derived from the SB. This corresponds to the execution of a script that governs the actions at the sub-array level. The tasks or script to be executed via the Scripting Environment are extracted from the Operation Scripts Repository. The Sub-array Sequencer executes a sequence of steps and synchronizes the actions of the individual telescopes. For this, it uses the abstraction services of telescopes. The Sub-array Sequencer is also responsible for steering any non-telescope-related components required to support the subarray action. Figure 5 illustrates the functional diagram of Sub-array Sequencer script execution.

- Non-telescope Sequencer: The Non-telescope Sequencers control those non-telescope related hardware assemblies that are not directly involved in sub-array actions (such as all-sky monitoring device). Such devices are not associated with any particular telescope may need to be controlled outside of the context of any particular sub-array action. Non-telescope sequencers are supervised by the Root Supervisor of the Resource Manager.

- Telescope Abstraction: The Telescope Abstraction provides a convenient interface to be used by the Sub-array Sequencer. It makes it easy to deal with one individual telescope. The prime focus here is uniformity of access across telescope types. 
- Observation Script Repository: The Observation Script Repository stores the different observation scripts that define the observation tasks to be performed. At its simplest level this is a directory location in the system used by the sequencer to retrieve the observation scripts. Eventually, the Observation Script Repository will be implemented as a database or script repository.

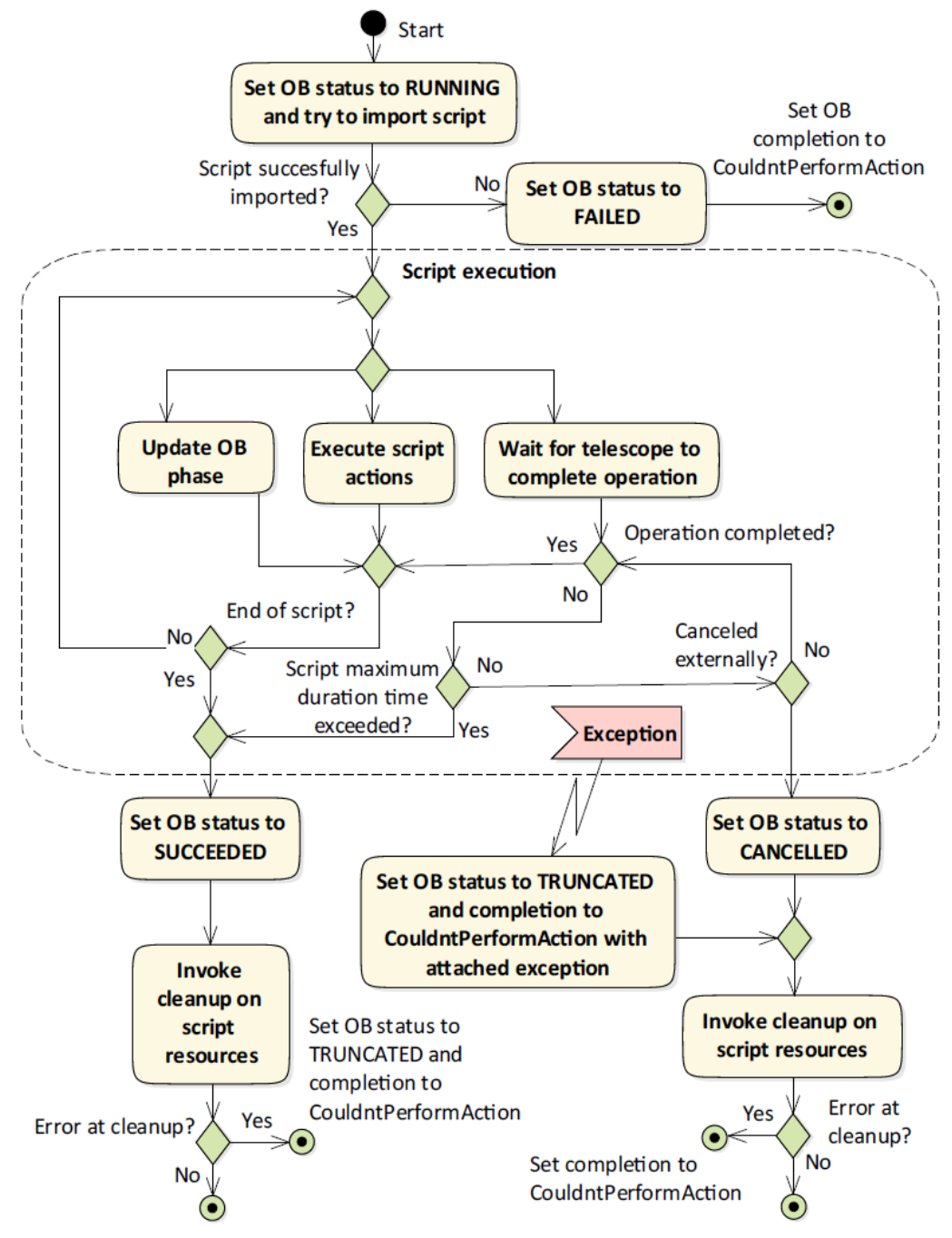

Figure 5. Sub-array Sequencer script execution. Each SB can consist of multiple observation segments with different observation parameters (e.g. coordinates), named Observation Blocks (OB).

\subsection{Scripting Environment}

The scripting environment provides the means to execute the main observatory operation modes. These are implemented as high-level Python scripts, and are executed by the Sub-array Sequencer component. This environment simplifies the script code by specifying a standard way of structuring the scripts and provides ways to test them. Most observation operations follow a standard set of phases (i.e. telescope camera configuration, slewing of telescopes, data acquisition, etc.). The scripting environment therefore allows a script to be defined in terms of phases. These detail conditions for completing a phase and moving on to the next one, or for running phases in parallel. The latter enables reduction of the configuration time and an increase of data acquisition time. The scripting environment supports the definition of commands for common operations shared between various operation modes. It provides a convenient access to the telescope and other resources, where definitions are imported automatically based on context. An example of a highlevel Python script is shown in Figure 6. 


\subsection{Sub-array Sequencer Runner}

To simplify testing of the scripts, the script environment provides a Sub-array Sequencer Runner terminal application. The latter is used to execute scripts without the need to run an ACS system instance with the CC and telescope resources. The operation mode, parameters and resources are provided via a JSON configuration file. When the script issues commands to the telescopes and other resources, predefined simulators/mock-ups of those resources are utilized. The observation state and status of simulators during the script execution is logged and displayed in the terminal.

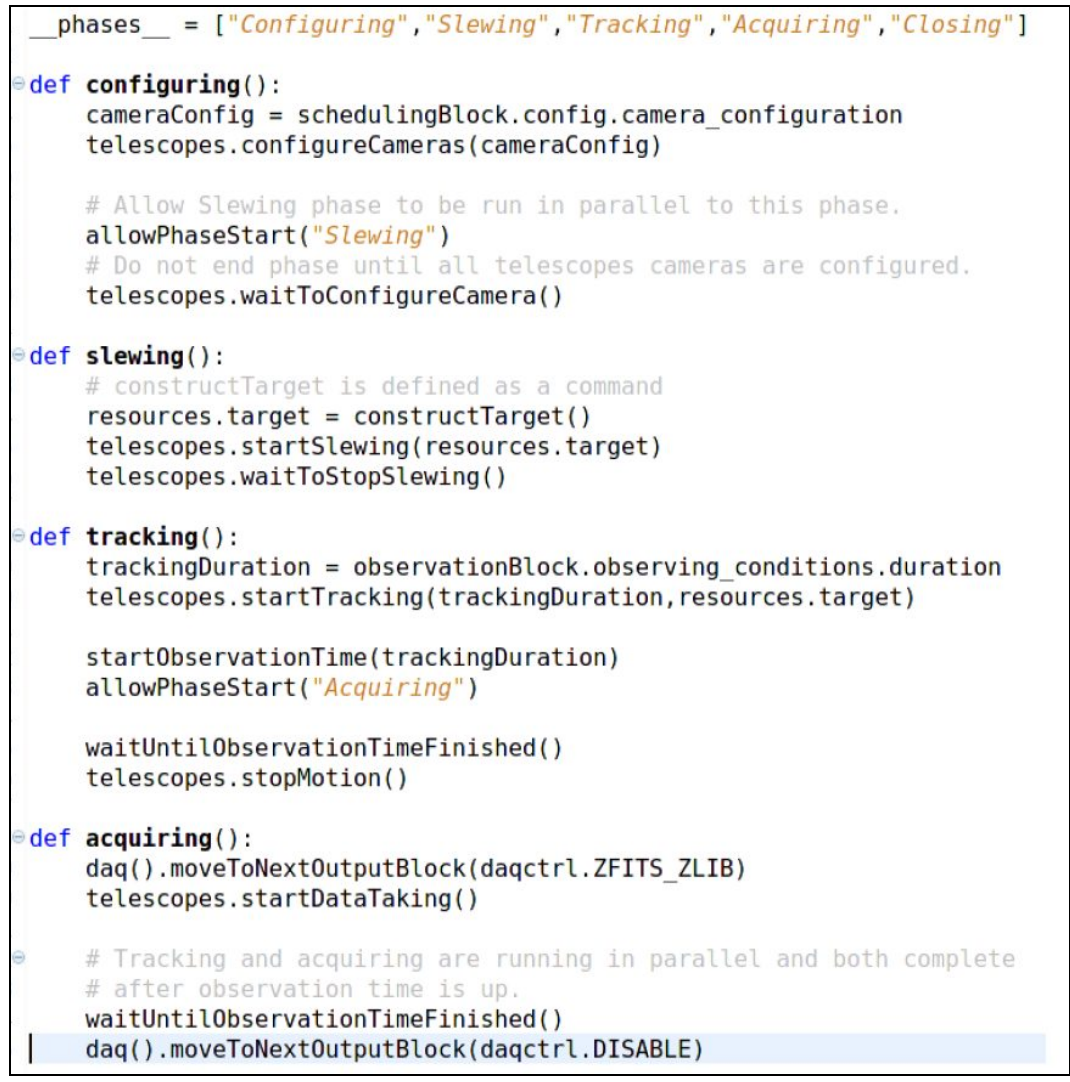

Figure 6. Example of a high-level sub-array sequencer script (written in Python).

\section{CONCLUSIONS}

In this paper, we have presented the main design features and the current implementation status of the MCC system. This prototype was intended as a proof of concept, applying a model-driven approach toward the development of the OES. The current version of the MCC system is capable of executing basic observation modes. It has been successfully integrated with the Short-Term Scheduler and UI sub-systems. Our plans for development in the near future include the following tasks:

- Complete the Resource Manager component, supervision tree and control ticket algorithm.

- Extend the scripting environment, to support a full set of CTA observatory operation modes, in particular those modes that handle target of opportunity ${ }^{* *}$ (ToO) events.

- Expand functionality, performance and stability tests of the system.

- Design and implement a Scripts Repository environment to handle high-level Python scripts submitted by user.

- Continue integration efforts with other OES sub-systems.

${ }^{* *}$ Target of Opportunity Proposals - https://www.eso.org/sci/observing/policies/too_policy.html 


\section{ACKNOWLEDGMENTS}

We gratefully acknowledge financial support from the agencies and organizations listed here: www.ctaobservatory.org/consortium_acknowledgments

\section{REFERENCES}

[1] Acharya, B., Actis, M., et al., "Introducing the CTA concept," Astroparticle Physics 43, 3 (2014).

[2] Pohl, K., Hoenninger, H., Achatz, R., and (Eds.), M. B., [Model-Based Engineering of Embedded Systems: The SPES 2020 Methodology], Springer Publishing, Berlin Heidelberg (2012).

[3] Oya, I., Füßling, M., et al., "The Software Architecture to Control the Cherenkov Telescope Array," in [Society of Photo-Optical Instrumentation Engineers (SPIE) Conference Series], Proc. SPIE 9913, 991303 (2016).

[4] Chiozzi, G., Jeram, B., Sommer, H., et al., "The ALMA common software: a developer-friendly CORBAbased framework," in [Observatory Operations: Strategies, Processes, and Systems], Proc. SPIE 9149, 91490H (2014).

[5] Schwarz, J., Sommer, H., and Farris, A., "The ALMA Software System," in [Analysis Software and Systems, XIII], Ochsenbein, F., Allen, M., and Egret, D., eds., ASP Conference Series 314, 634-652 (2004).

[6] Füssling, M., Oya, I., et al., "Status of the array control and data acquisition system for the Cherenkov Telescope Array," in [Society of Photo-Optical Instrumentation Engineers (SPIE) Conference Series], Proc. SPIE 9913, 99133C (2016)

[7] Colomé, J., Colomer, P., Campreciós, J., et al., "Artificial intelligence for the CTA Observatory scheduler," in [Observatory Operations: Strategies, Processes, and Systems V], Proc. SPIE 9149, 91490H (Aug. 2014).

[8] Lyard, E., Walter, R., Kosack, K., et al., "Modern middleware for the data acquisition of the Cherenkov Telescope Array," in [Proceedings of the 34th International Cosmic Ray Conference (ICRC2015), The Hague, The Netherlands], (2015).

[9] Sadeh, I., Oya, I., Pietriga, E., et al., "Prototyping the graphical user interface for the operator of the Cherenkov Telescope Array," in [Society of Photo-Optical Instrumentation Engineers (SPIE) Conference Series], Proc. SPIE 9913, 99130X (2016) 